\title{
Post-surgery severe hypocalcemia in primary hyperparathyroidism preoperatively treated with zoledronic acid
}

\author{
Salvatore Maria Corsello, Rosa Maria Paragliola, Pietro Locantore, Francesca Ingraudo, \\ Maria Pia Ricciato, Carlo Antonio Rota, Paola Senes, Alfredo Pontecorvi
}

Department of Endocrinology, Catholic University School of Medicine, Largo A. Gemelli, Rome, Italy

\begin{abstract}
Zoledronic acid is a newly FDA-approved bisphosphonate for the treatment of hypercalcemia of malignancy. Although the safety and efficacy of this drug in treating hypercalcemia associated with hyperparathyroidism have not yet been established in clinically controlled trials, its off-label use is not uncommon. We describe a patient with primary hyperparathyroidism treated with zoledronic acid who developed severe postoperative hypocalcemia. A 64-yr-old woman was admitted with severe hypercalcemia. She was treated with rehydration, calcitonin, methylprednisolone, furosemide as well as $\mathbf{4 m g}$ /day of zoledronic acid for two consecutive days. Primary hyperparathyroidism caused by a right inferior parathyroid lesion was diagnosed. While awaiting surgery, she continued furosemide, methylprednisolone and hydration: after one week, serum calcium had fallen to such a low level that a short-term calcium carbonate supplementation was required. Three weeks after admission, the patient underwent selective right inferior parathyroidectomy, followed by reduction of PTH. During the postoperative period the patient presented severe hypocalcemia resistant to the usual treatment. Serum calcium levels returned to normal three months after surgery. The severity of hypocalcemia and the resistance to conventional treatments suggest that the effect of hungry bone syndrome could be worse in patients treated with bisphosphonates in the preoperative phase.
\end{abstract}

Key words: Bisphosphonates, Hungry bone syndrome, Hypercalcemia, Hyperparathyroidism, Hypocalcemia, Parathyroidectomy, Parathyroid hormone, Zoledronic acid

\section{INTRODUCTION}

Primary hyperparathyroidism $(\mathrm{PH})$ is caused by an excess of parathyroid hormone (PTH) secretion

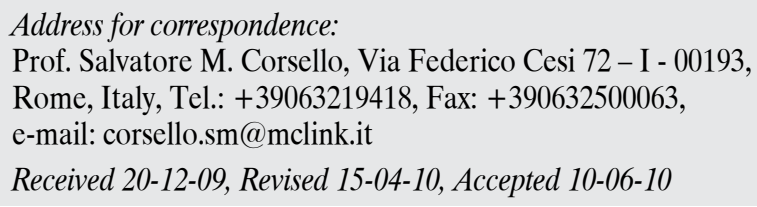

from one or more parathyroids, provoking an alteration in the calcium-phosphorus balance. ${ }^{1} \mathrm{~A}$ few patients with $\mathrm{PH}$ develop parathyroid crisis, which is characterized by severe hypercalcemia with a serum calcium concentration above $3.75 \mathrm{mmol} / \mathrm{l}$, and certain characteristic clinical features of hypercalcemia, in particular, central nervous system dysfunction. This situation represents an endocrine emergency. Hy- 
percalcemia should be pharmacologically corrected and kept under control while waiting for surgery. The strategies preferred for the medical treatment of these cases are hydration, furosemide (to increase calcium excretion) and corticosteroids. ${ }^{2}$ In addition, pamidronate is administrated to decrease bone resorption and lower the serum calcium concentration within 48 to 72 hours. Zoledronic acid, a newer bisphosphonate (BP) which is FDA-approved for the treatment of hypercalcemia of malignancy, could also be considered in these cases.

Surgery represents the treatment of choice in symptomatic patients with PH. Parathyroidectomy is effective for restoring normocalcemia and longterm normal parathyroid function in the majority of patients.

In some cases, however, the postoperative hypocalcemia is severe and prolonged despite normal or even elevated levels of PTH. This phenomenon, called "hungry bone syndrome", most often occurs in patients who have developed bone disease preoperatively as a result of chronic increase in bone resorption induced by high levels of PTH (osteitis fibrosa). ${ }^{3}$

We report a patient who was brought to the Emergency Unit of our hospital with severe hypercalcemia, treated by a therapeutic regimen which included zoledronic acid, and showed severe hypocalcemia after parathyroid surgery.

\section{CASE REPORT}

A 64-yr-old woman was admitted to the Emergency Unit for an accidental fall causing a contusive trauma to her knees. On examination she was alert but disoriented as to time and space. Chest and neurological examinations were negative. Computed tomography scan of the brain did not demonstrate any hemorrhage. Blood pressure was 125/70 $\mathrm{mmHg}$ and heart rate was 80 beats/min. Blood samples were taken and the results of various determinations are as follows: hemoglobin: $12.3 \mathrm{~g} / \mathrm{dl}$; white blood cells: $13250 \times 10^{9} / 1$; glycemia: $4.88 \mathrm{mmol} / \mathrm{l}$; Blood Urea Nitrogen (BUN): $43 \mathrm{mg} / \mathrm{dl}$; sodium: $125 \mathrm{mEq} / \mathrm{l}$; potassium: $2.3 \mathrm{mEq} / \mathrm{l}$; magnesium: $1.9 \mathrm{mg} / \mathrm{dl}$; lactate dehydrogenase: $587 \mathrm{U} / 1$; troponin T: $0.08 \mathrm{U} / \mathrm{l}$; creatine kinase: $64 \mathrm{U} / \mathrm{l}$; alkaline phosphatase: $1258 \mathrm{U} / \mathrm{l}$. The most relevant finding was a severe hypercalcemia [5 mmol/1, (2.12-2.62)]. Therefore, a rehydration therapy with potassium supplementation was initiated along with intravenous (IV) calcitonin $400 \mathrm{IU} /$ day in $500 \mathrm{ml}$ normal saline solution, IV methylprednisolone $40 \mathrm{mg} 2$ times/day, IV furosemide $40 \mathrm{mg} /$ day, while IV zoledronic acid $4 \mathrm{mg} /$ day in $100 \mathrm{ml}$ normal saline solution was given for two consecutive days.

After a 24-hour observation, serum calcium was $3.67 \mathrm{mmol} / \mathrm{l}$ and PTH levels were extremely elevated (2357 ng/l, n.v. 10-65), while plasma levels of 25hydroxyvitamin $\mathrm{D}$ were low $[<18.72 \mathrm{nmol} / \mathrm{l}$, (n.v. 18.72-102.3)].

Neck ultrasound (US) disclosed a $25 \mathrm{~mm}$ ovalshaped mass connected with the right inferior parathyroid gland. Parathyroid scintigraphy $\left({ }^{99 \mathrm{~m}}\right.$ technetium - sestamibi $+{ }^{99 \mathrm{~m}}$ technetium - pertechnetate) demonstrated an area of uptake localized in the inferior part of the right lobe of the thyroid gland, concordant with the US finding. Appropriate endocrine tests excluded multiple endocrine neoplasia (MEN) 1 and 2A. Selective right inferior parathyroidectomy was planned. While awaiting surgery, the patient continued the therapy with IV furosemide $40 \mathrm{mg} /$ day, IV methylprednisolone $40 \mathrm{mg} /$ day and hydration, which resulted in a further progressive reduction of serum calcium concentration. After one week, the serum calcium values had fallen to $1.92 \mathrm{mmol} / \mathrm{l}$ and a short-term oral calcium carbonate supplementation ( $1 \mathrm{~g} /$ day) was required.

Three weeks after admission, the patient underwent selective right inferior parathyroidectomy. Intraoperative PTH monitoring showed a significant reduction of PTH levels (from basal levels of $2876 \mathrm{ng} / \mathrm{l}$ to 154 ng/l 20 minutes after resection). The preoperative suspicion of parathyroid adenoma was confirmed by histological examination.

During the postoperative period, the patient presented severe hypocalcemia which was resistant to the usual treatment with IV calcium gluconate and oral calcitriol, so that a daily clinical and laboratory monitoring in hospital was required. The therapy was adjusted to a regimen of IV calcium gluconate 6-8 $\mathrm{g} /$ day (= calcium 540-720 mg/day), oral calcitriol 3 $\mu \mathrm{g} /$ day and transient use of IV calcitriol $1.5 \mu \mathrm{g} /$ day, and normal values of serum calcium were reached 


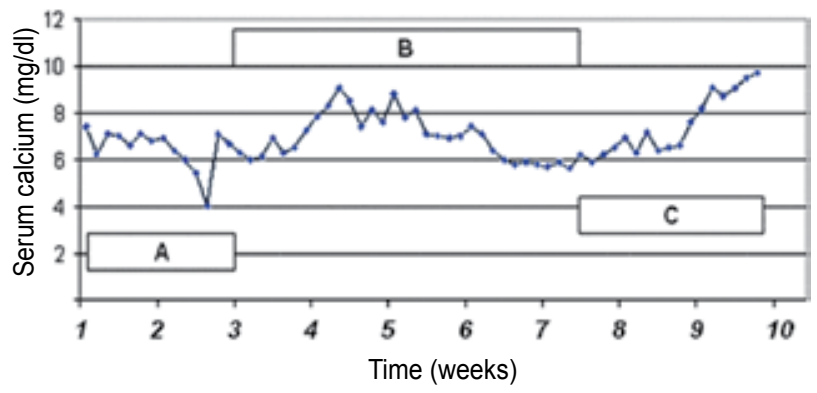

Figure 1. Postoperative serum calcium monitoring, related to the daily therapeutic scheme. A. IV calcium gluconate $3 \mathrm{~g}$; oral calcitriol $1.5 \mu \mathrm{g}$, B. IV calcium gluconate $6 \mathrm{~g}$; oral calcitriol 1.5 $\mu \mathrm{g}$, C. IV calcium gluconate $6-8 \mathrm{~g}$; oral calcitriol $3 \mu \mathrm{g}$; IV calcitriol $1.5 \mu \mathrm{g}$. IV: intravenous.

in three months after surgery (Figure 1). During the postoperative period, $\mathrm{PTH}$ values remained very low ( $10 \mathrm{ng} / \mathrm{l})$, while alkaline phosphatase values were consistently high ( 1000 U/l).

The patient was discharged on oral calcitriol 3 $\mu \mathrm{g} /$ day, which was progressively reduced and discontinued after three months.

\section{DISCUSSION}

BPs are a class of drugs that are composed of a basic phosphate-carbon-phosphate core and bind avidly to the bone mineral at sites of active bone metabolism. Their selective action on bone tissue is due to their high affinity for mineralized bone. In fact, these compounds have high affinity for calcium ions and therefore target bone mineral, where they are internalized by bone-resorbing osteclasts and inhibit osteoclast function. BPs can be divided into two classes: nitrogen containing (N-BPs), such as zoledronic acid, pamidronate, alendronate, ibandronate and risedronate that are the more potent, and non-nitrogen containing (non N-BPs). ${ }^{4} \mathrm{~N}$-BPs act intracellulary by inhibiting farnesyl diphosphate synthase, an enzyme of the mevalonate pathway. Several signaling molecules in this pathway are involved in the regulation of cell proliferation, cell survival and cytoskeletal organization. ${ }^{5}$

Zoledronic acid is a III generation N-BP that inhibits osteoclast-mediated bone resorption, inducing osteoclast apoptosis without interfering with bone formation, mineralization or mechanical properties.
Some studies in animals treated with zoledronic acid have demonstrated a possible beneficial effect on the capacity of bone for regeneration. ${ }^{6}$

Zoledronic acid is FDA-approved for the treatment of hypercalcemia of malignancy and bone metastases from any solid tumor, but its off-label use in hypercalcemia associated with $\mathrm{PH}$ is not uncommon.

In the present report we describe the case of a patient with $\mathrm{PH}$, treated with zoledronic acid for serious hypercalcemia, who developed postoperatively very severe hypocalcemia. This situation can be attributed to the hungry bone syndrome, which is responsible for some cases of severe hypocalcemia after parathyroidectomy. This syndrome occurs in patients who have developed an increased bone resorption during the preoperative phase, induced by high PTH levels. In PH the increase of osteoclast activity causes a bone resorption associated with fibrosis, further proliferation of osteoclasts and hemosiderin macrophages (osteitis fibrosa). The removal of the $\mathrm{PTH}$ excess reduces the bone resorptive activity. The increased bone formation continues for at least several weeks, resulting in massive skeletal deposition of calcium causing hypocalcemia, hypophosphatemia and tetany. Within a few weeks, the low serum calcium leads to increase in PTH secretion from the normal glands, sometimes to abnormally high levels. The hypocalcemia is subsequently corrected by the mobilization of calcium from the skeleton and the reduction of urinary calcium excretion. Through these mechanisms the hypocalcemia of the hungry bone syndrome is usually self-limited. The hungry bone syndrome is rare today, but it should probably not be underestimated by clinicians.

In patients who undergo surgery for $\mathrm{PH}$, predictive factors for the risk of developing hungry bone syndrome are: adenoma size, preoperative BUN concentration, preoperative alkaline phosphatase concentration and age, while preoperative levels of serum calcium and PTH do not appear to be predictive. ${ }^{3}$ Recently, an association between vitamin D deficiency and hungry bone has been proposed. In fact, patients with vitamin D-deficiency undergoing parathyroidectomy are at increased risk of postoperative hypocalcemia and hungry bone syndrome, which underscores the importance of preoperative 
assessment of vitamin D status in all patients with PH. ${ }^{7}$ In our patient, preoperative vitamin D levels, evaluated as plasma 25 hydroxyvitamin $\mathrm{D}$, were low in accord with PTH-induced 25 hydroxyvitamin D-1 $\alpha$ hydroxylase activity.

The treatment of hungry bone aims at correcting abnormalities such as hypocalcemia, hypomagnesemia and hypophosphatemia. In our case, the postoperative clinical picture was particularly severe and resistant to usual therapies. It seems relevant that, in the immediate preoperative phase, this patient was treated with a long-acting BP (zoledronic acid) to correct the serious hypercalcemia.

In the literature, the causal relationship between $\mathrm{BP}$ use and post operative hungry bone syndrome is still a topic of controversy. Some authors maintain that the administration of BPs in $\mathrm{PH}$ can prevent the occurrence of hungry bone syndrome after parathyroidectomy, suggesting the possibility of preoperative treatment with pamidronate. ${ }^{8}$ On the other hand, Graal et al reported a severe and long-standing hypocalcemia which developed after parathyroidectomy "despite" pre-treatment with IV pamidronate and vitamin D supplementation. ${ }^{9}$ Whitson et al described an example of hungry bone syndrome in a patient with extensive Paget disease of the bones who, after receiving risedronate, showed an acute suppression of bone resorption, while elevated bone formation rates continued. ${ }^{10} \mathrm{~A}$ recent report described the first case of hungry bone syndrome induced by therapy based on Cinacalcet, a calcimimetic agent approved for the treatment of secondary hyperparathyroidism. ${ }^{11,12}$

It is suggested that the hypocalcemia in our patient occurred because BP therapy effectively reduced osteoclastic bone resorption, while osteoblastic bone formation persisted. In other words, the failure to correct the postoperative hypocalcemia was due to the intense anti-resorptive action of zoledronic acid in bone which blocked the mobilization of calcium from the skeleton. The failure of serum PTH to rise postoperatively was probably a contributing factor by allowing an unopposed bisphosphonate action.

\section{CONCLUSION}

To our knowledge, this patient represents the first reported case of severe postoperative hypocalcemia possibly related to the preoperative use of zoledronic acid. A parathyroid crisis associated with primary hyperparathyroidism is an endocrine emergency requiring a quick medical approach. Because of a life risk situation, it is reasonable in such cases to consider a treatment which may not yet be conventionally approved for patients with primary hyperparathyroidism. Given the widespread off-label use of this potent and long-acting drug to manage severe hypercalcemia in patients with hyperparathyroidism while awaiting surgery, physicians should be made aware of the potential risk of severe and prolonged hypocalcemia, which may expose patients to a life-threatening situation after surgery.

\section{SOURCES OF SUPPORT}

No grants have been received.

\section{DISCLOSURE INFORMATION}

None of the authors has any relationship with products or companies mentioned in or related to the subject matter of the present article.

\section{REFERENCES}

1. Bilezikian JP, Silverberg SJ, 2000 Clinical spectrum of primary hyperparathyroidism. Rev Endocr Metab Disord 1: 237-245.

2. Makras P, Papapoulos SE, 2009 Medical treatment of hypercalcaemia. Hormones (Athens) 8: 83-95.

3. Brasier AR, Nussbaum SR, 1988 Hungry bone syndrome: clinical and biochemical predictors of its occurrence after parathyroid surgery. Am J Med 84: 654-60.

4. Benford HL, Frith JC, Auriola S, Mönkkönen J, Rogers MJ, 1999 Farnesol and geranylgeraniol prevent activation of caspases by aminobisphosphonates: biochemical evidence for two distinct pharmacological classes of bisphosphonate drugs. Mol Pharmacol 56: 131-140.

5. Luckman SP, Hughes DE, Coxon FP, Graham R, Russell G, Rogers MJ, 1998 Nitrogen-containing bisphosphonates inhibit the mevalonate pathway and prevent post-translational prenylation of GTP-binding proteins, including Ras. J Bone Miner Res 13: 581-589.

6. Bilston LE, Little DG, Smith NC, Williams P, Briody J, 2002 Zoledronic acid improves the mechanical properties of normal and healing bone. Clin Biomech (Bristol, Avon) 17: 716-718.

7. Silverberg SJ, 2007 Vitamin D deficiency and primary hyperparathyroidism. J Bone Min Res 22: Suppl 2: V100-104. 
8. Lee IT, Sheu WH, Tu ST, Kuo SW, Pei D, 2006 Bisphosphonate pretreatment attenuates hungry bone syndrome postoperatively in subjects with primary hyperparathyroidism. J Bone Min Metab 24: 255-258.

9. Graal MB, Wolffenbuttel BH, 1998 Consequences of long-term hyperparathyroidism. Neth J Med 53: 3742.

10. Whitson HE, Lobaugh B, Lyles KW, 2006 Severe hypocalcemia following bisphosphonate treatment in a patient with Paget's disease of bone. Bone 39: 954958.

11. Lazar ES, Stankus N, 2007 Cinacalcet-induced hungry bone syndrome. Semin Dial 20: 83-85.

12. Yavropoulou MP, Kotsa K, Gotzamani-Psarrakou A, et al, 2010 Cinacalcet in hyperparathyroidism secondary to $\mathrm{X}$-linked hypophosphatemic rickets: case report and brief literature review. Hormones (Athens) 9: 274-278. 